\title{
The many tunes of perisomatic targeting interneurons in the hippocampal network
}

\author{
Tommas J. Ellender ${ }^{1,2}$ and Ole Paulsen ${ }^{1,3 *}$ \\ OXION Initiative, Department of Physiology, Anatomy and Genetics, University of Oxford, Oxford, UK \\ 2 Medical Research Council Anatomical Neuropharmacology Unit, University of Oxford, Oxford, UK \\ 3 Physiological Laboratory, Department of Physiology, Development and Neuroscience, University of Cambridge, Cambridge, UK
}

\section{Edited by:}

Yehezkel Ben-Ari, Institut National de la

Santé et de la Recherche Médicale,

France

\section{Reviewed by:}

Alain Destexhe, Unité de

Neurosciences, Information and

Complexité Centre National de la

Recherche Scientifique, France

Barry W. Connors, Brown University,

USA

Rustem Khazipov, Institut National de

la Santé et de la Recherche Médicale,

France

Krecho Krnkevic, McGill University,

Canada

\section{*Correspondence:}

Ole Paulsen, Physiological Laboratory,

Department of Physiology,

Development and Neuroscience,

University of Cambridge, Downing

Street, Cambridge CB2 3EG, UK.

e-mail:op210@cam.ac.uk
The axonal targets of perisomatic targeting interneurons make them ideally suited to synchronize excitatory neurons. As such they have been implicated in rhythm generation of network activity in many brain regions including the hippocampus. However, several recent publications indicate that their roles extend beyond that of rhythm generation. Firstly, it has been shown that, in addition to rhythm generation, GABAergic perisomatic inhibition also serves as a current generator contributing significantly to hippocampal oscillatory EEG signals. Furthermore, GABAergic interneurons have a previously unrecognized role in the initiation of hippocampal population bursts, both in the developing and adult hippocampus. In this review, we describe these new observations in detail and discuss the implications they have for our understanding of the mechanisms underlying physiological and pathological hippocampal network activities. This review is part of the Frontiers in Cellular Neuroscience's special topic entitled "GABA signaling in health and disease" based on the meeting at the CNCR Amsterdam.

Keywords: inhibition, GABA, perisomatic targeting interneuron, hippocampus, network oscillation, gamma oscillation, sharp wave-ripple, population burst

\section{INTRODUCTION}

Like many cortical structures, the hippocampal CA3 region consists of a large network of rather uniform, recurrently connected, excitatory neurons together with a smaller but diverse population of inhibitory neurons (Freund and Buzsaki, 1996; Vizi and Kiss, 1998; Klausberger and Somogyi, 2008). This interconnected network of excitatory and inhibitory neurons enables the CA3 circuitry to generate a wide range of network activities depending on its inputs and the state of the network. These network states have been ascribed important roles in hippocampal function, such as spatial navigation (O’Keefe and Dostrovsky, 1971; Huxter et al., 2003), memory encoding and retrieval (Lisman and Idiart, 1995; Jensen and Lisman, 1996), and memory consolidation (Buzsaki, 1986, 1989). Understanding the mechanisms by which these different network patterns are generated might shed light on the underlying computational principles by which they perform their functions. Many studies have indicated an important role of GABAergic inhibition in generating these patterns (Buzsaki et al., 1983; Ylinen et al., 1995; Csicsvari et al., 2003).

Several hippocampal slice preparations have been created which generate similar network activity to that seen in vivo (Ben-Ari et al., 1989; Fisahn et al., 1998; Kubota et al., 2003). These in vitro preparations enable investigation of network activity using extracellular field recording in combination with fast exchange of pharmacological agents, visually guided patch-clamp recordings and advanced imaging techniques (Hajos et al., 2009).
In this review, we discuss several recent papers that reveal two previously unrecognized roles of GABAergic transmission in hippocampal network activity, as seen in field recordings. Firstly, we discuss the finding that the local field potential (LFP) reflects more of inhibitory currents than previously thought. Secondly, we discuss the finding that some GABAergic neurons can initiate hippocampal population bursts, not only early in development, but, unexpectedly, also in the mature CA3 network. These findings emphasize the increasing number of roles ascribed to interneurons in the generation of physiological hippocampal network activity. We also briefly discuss the potential implications of these findings for our understanding of the mechanisms underlying pathological network activity (see Table $\mathbf{1}$ ).

\section{HIPPOCAMPAL OSCILLATIONS}

Many types of hippocampal network oscillations are driven by inhibitory neurons. Here we discuss the recent finding that inhibitory events not only generate the rhythm of these oscillations, but do in fact also contribute directly to the recorded oscillatory EEG signal. For the purpose of this review we focus mainly on perisomatic targeting interneurons, although dendritic targeting interneurons most likely also play an important role in oscillogenesis. For a recent review on the family of dendritic targeting interneurons and hippocampal network activity, see Klausberger (2009). 


\section{RHYTHM GENERATION}

The involvement of interneurons in rhythm generation has been well documented. For theta oscillations $(4-8 \mathrm{~Hz})$, for example, it is thought that oscillations in individual cells (Alonso and Llinas, 1989; Strata, 1998; Buzsaki, 2002) are stabilized and synchronized by a set of inhibitory feedback circuits (Buzsaki et al., 1983; Leung and Yim, 1986; Soltesz and Deschenes, 1993; Cobb et al., 1995; Klausberger et al., 2003), as recently shown in an intact isolated hippocampus in vitro (Goutagny et al., 2009). In addition, external GABAergic inputs arriving from the medial septum and brainstem (Petsche and Stumpf, 1962; Stewart and Fox, 1990) could also contribute to rhythm generation, potentially serving as a pacemaker for the theta rhythm (Stewart and Fox, 1990).

Gamma oscillations $(30-100 \mathrm{~Hz})$ are commonly observed nested within theta oscillations in the hippocampus (Bragin et al., 1995; Buzsaki et al., 2003; Csicsvari et al., 2003). Studies into the mechanisms underlying carbachol-induced gamma oscillations in hippocampal slices have indicated an important role for perisomatic inhibition in rhythm generation (Fisahn et al., 1998; Mann et al., 2005; Oren et al., 2006). In particular, it was observed that prolongation of the $\mathrm{GABA}_{\mathrm{A}}$ receptor-mediated inhibitory postsynaptic currents (IPSCs) with barbiturate reduces the frequency of gamma oscillations (Fisahn et al., 1998). Modeling studies also implicate inhibitory interneurons in rhythm generation (Wang and Buzsaki, 1996; Traub et al., 1997; Brunel and Wang, 2003), as do in vivo recordings (Penttonen et al., 1998; Tukker et al., 2007). For a discussion of in vitro gamma oscillation models in relation to in vivo activity, see Hajos and Paulsen (2009).

Although the rhythm generation of many oscillations has been extensively studied, little is known about the currents underlying the observed field events. Field activity is generated by the sum of currents flowing into and out of cells. It has long been thought that excitatory currents contribute predominantly to the recorded field events (see Figures 1A,B). Several recent papers have now challenged this assumption and shown that inhibition, especially arising from perisomatic targeting interneurons, can contribute significantly to the current generation (see Figures 1A,C).

A

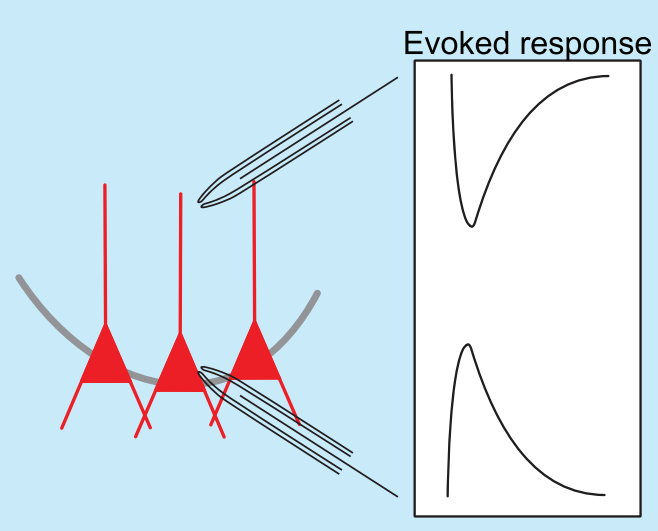

Evoked response Oscillation

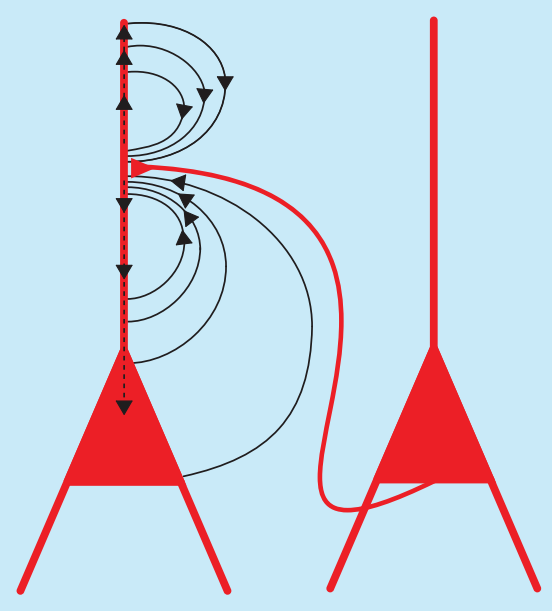

C
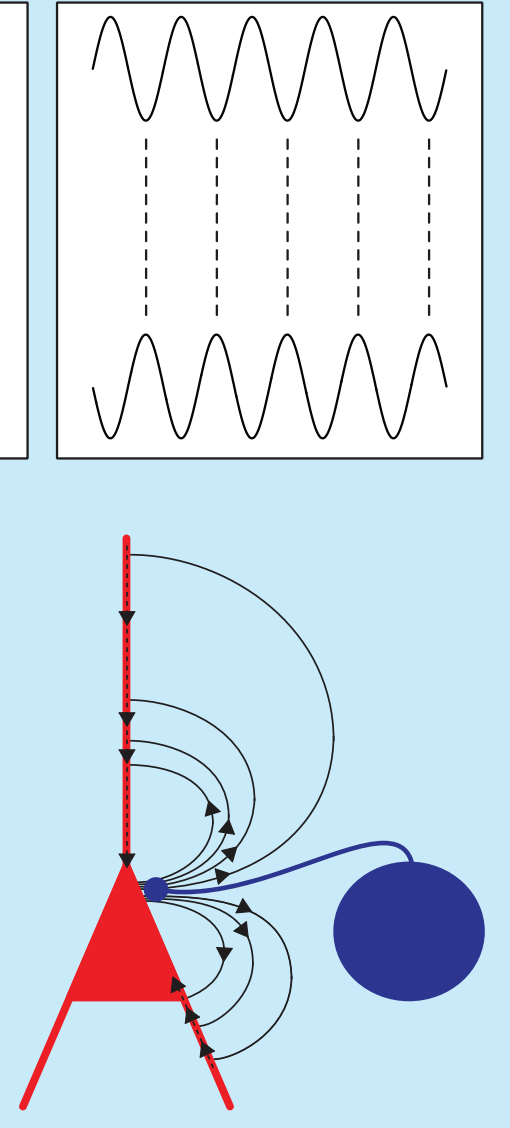

FIGURE 1 | Schematic diagram of hippocampal local field potentials and the underlying current sinks and sources. (A) Recording electrodes placed in the dendritic and somatic regions of the hippocampus show a phase reversal for both evoked responses and oscillatory activity (B) Excitatory synaptic events at the apical dendrite of pyramidal neurons generate an active current sink as positive ions flow into neurons: a concurrent passive source is recorded from the somatic region. (C) Inhibitory synaptic events at the perisomatic regions of pyramidal neurons generate an active source accompanied by a passive current sink at the dendrites. 
Table 1 | Involvement of perisomatic targeting interneurons in hippocampal network activity.

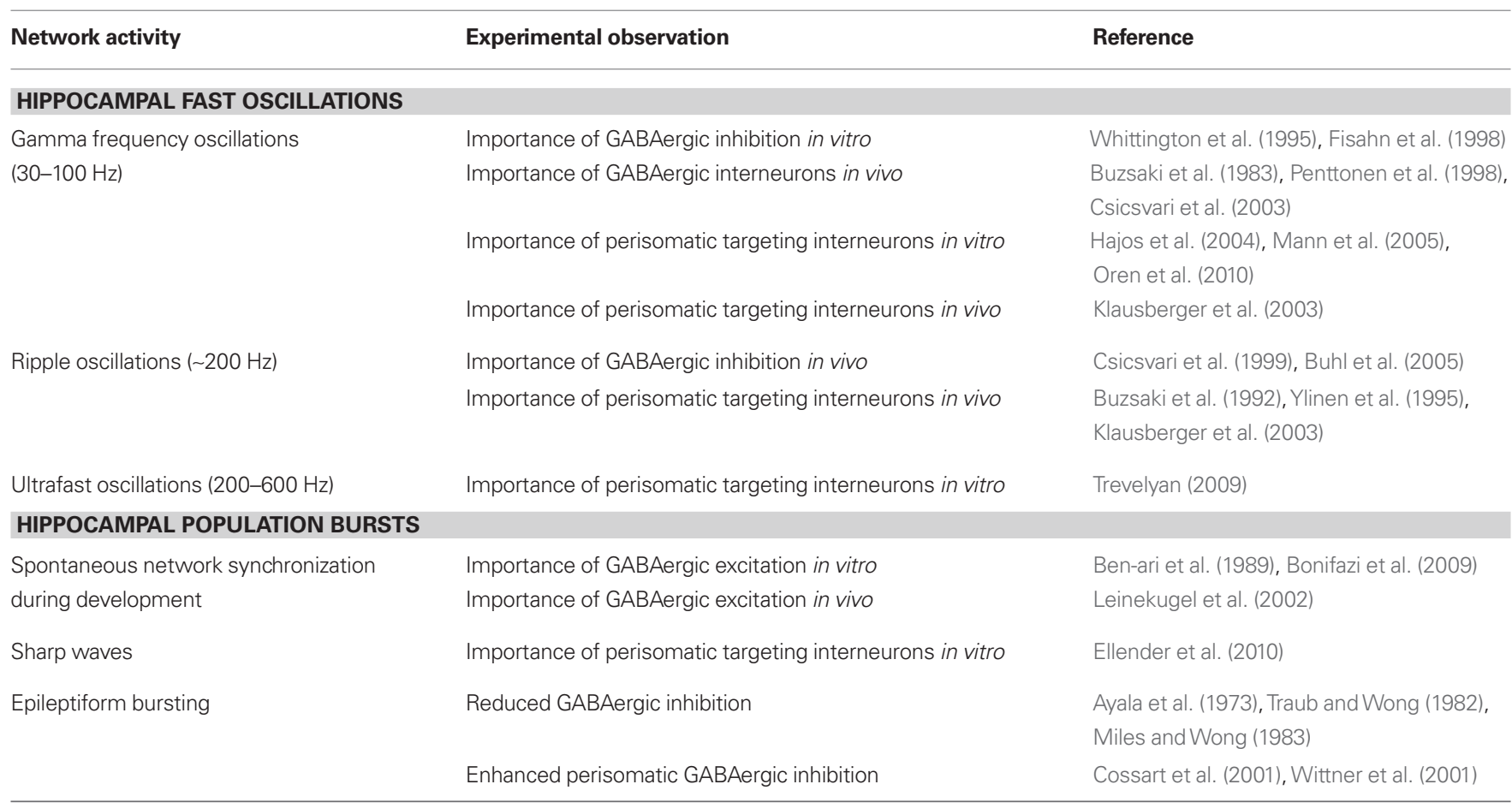

\section{CURRENT GENERATION}

A recent paper by Oren et al. (2010) analyzed the contribution of excitatory and inhibitory synaptic currents, as well as spiking activity of CA3 hippocampal neurons, to the LFP in carbachol-induced gamma oscillations. It was observed that gamma oscillation amplitude fluctuated over time. Changes in the oscillation amplitude were quantified by the amplitude of the wavelet transform. By correlating the amplitude of the oscillation as seen in the field and the activity in individual cells it was suggested that the largest contributor to the field was the inhibitory currents in excitatory neurons, with a smaller contribution from the spiking activity of pyramidal neurons. Three lines of evidence support this conclusion. Firstly, the mean cycle amplitude was significantly higher when perisomatic interneurons discharged. There was no such direct relationship between the discharge of a pyramidal neuron and the LFP amplitude, although a small contribution was found for the slow action currents, resulting from the activation of a repolarization conductance, to the early component of the LFP. Secondly, the amplitude of the LFP signal correlated significantly with the inhibitory synaptic currents, whereas no such correlation was seen with excitatory synaptic currents. Thirdly, the inhibitory events recorded from pyramidal neurons were highly synchronized with the LFP.

Direct evidence that inhibitory neurons can produce significant field events was provided in a recent study by Glickfeld et al. (2009), who investigated whether activity of single anatomically identified interneurons is inhibitory or excitatory for downstream targets, using a combination of single cell stimulation and LFP recording. Surprisingly, activity in a single interneuron generated a transmembrane current sufficiently large to be reflected in the LFP recorded with an extracellular electrode (Glickfeld et al., 2009). The largest field event was seen following stimulation of perisomatic targeting interneurons (basket and axo-axonic cells). This observation was recently confirmed in a study by Bazelot et al. (2010), who also showed that single interneurons can generate a measurable field response. They showed that stimulation of a single perisomatic targeting interneuron can generate a field response of $\sim 30 \mu \mathrm{V}$. If field IPSPs add linearly, a field recording of hippocampal gamma oscillations in a hippocampal slice in the range of $100-500 \mu \mathrm{V}$ (Fisahn et al., 1998) could be accounted for by the synchronous activity of approximately 10 perisomatic targeting interneurons.

\section{RIPPLE AND ULTRAFAST OSCILLATIONS}

The fact that perisomatic inhibition has a large contribution to gamma oscillations as measured in the field raises the possibility that they might also contribute to faster field oscillations, such as ripple oscillations as part of sharp wave-ripple complexes, or ultrafast oscillations, as seen in pathological states. The cellular basis of ripple oscillations is still a matter of debate, and several possible explanations have been proposed.

Firstly, it has been suggested that the synchronous depolarization of CA1 neurons by CA3 pyramidal neuron activity sets in motion a dynamic interaction between CA1 pyramidal cells and CA1 interneurons, resulting in an oscillatory field potential between 120 and $200 \mathrm{~Hz}$ as seen in the stratum pyramidale (Buzsaki et al., 1992). Occasionally, CA3 ripples were seen concurrent with those occurring in CA1, but the CA3 ripples were of a lower frequency $(80-140 \mathrm{~Hz}$ ) (Ylinen et al., 1995) and not correlated to unit activity in CA1. Combined, these results suggest that CA1 ripple oscillations emerge from the CA1 cell population rather than being a passive response to high frequency input from CA3 (Ylinen et al., 1995). The specific synaptic currents mediating ripple oscillations were suggested to be synchronized 
somatic IPSCs in CA1 pyramidal neurons (Ylinen et al., 1995). Potentially, the interneurons could be synchronized by gap junctions (Katsumaru et al., 1988) as halothane anesthesia abolishes ripple activity (Ylinen et al., 1995). Interestingly, the emergence of ripple oscillations coincides with the transition from depolarizing to hyperpolarizing GABA action during development, suggesting that a dynamic interaction between excitation and inhibition is needed for ripple oscillations to occur (Buhl and Buzsaki, 2005).

A second proposed mechanism for CA1 ripple oscillations involves a network of pyramidal neurons interconnected by axonal gap junctions (Draguhn et al., 1998). It was shown that the CA1 region of hippocampal slices could produce ripple oscillations that were not affected by block of both excitatory and inhibitory synaptic currents, but disappeared when gap junction blockers were used, although these compounds are notoriously unspecific (Connors and Long, 2004). However, in vivo, CA1 pyramidal neurons tend to burst at higher frequencies than ripple oscillations, which would not support this hypothesis (Csicsvari et al., 1999).

Thirdly, it has been suggested that ripples observed in CA1 are the extracellular reflection of the synchronous firing of small groups of pyramidal neurons. These "population spikes" could be the result of intrinsically generated pyramidal neuron spiking, synchronized by recurrent connections (Dzhala and Staley, 2004). These three possibilities are not mutually exclusive and a combination of them is possible.

Similar explanations have been forwarded for ultrafast oscillations seen during epileptiform activity (Le Van Quyen et al., 2006), including the idea of synchronized "population spikes" (Bragin et al., 2000, 2002, 2007), although desynchronized spiking has also been proposed (Foffani et al., 2007). It has been suggested that ripple activity is crucial for the development of an epileptic focus in the developing (Khalilov et al., 2005), and adult brain (Traub et al., 2001b; Grenier et al., 2003), whereas others have proposed that ripples might rather be protective and restrict epileptiform spread (Trevelyan et al., 2006, 2007). A recent paper investigated the correlation between the fast oscillations seen in the field during interictal events in a $0 \mathrm{Mg}^{2+}$ model of epileptiform activity and IPSCs recorded intracellularly from pyramidal neurons (Trevelyan, 2009). The author found that IPSCs recorded from closely located pyramidal neurons are highly synchronous, whereas IPSCs recorded from neurons located more than $200 \mu \mathrm{m}$ apart were not synchronous. The author used dual whole-cell recordings with one pyramidal neuron held in voltage-clamp and another in current-clamp combined with an extracellular electrode to record the field potential. Using sophisticated analysis combined with modeling a close correlation was seen between the high frequency oscillation (HFO) in the field and the intracellular recordings from the pyramidal neurons. This correlation was strongest in the preictal period and fell as the event developed into a full ictal event. The author argued that it is the transmembrane currents generated by perisomatic inhibition that underlies these HFOs (Trevelyan, 2009).

In summary, for both gamma and ripple oscillations, recent evidence suggests that IPSCs of perisomatic origin contribute to the oscillatory field event.

\section{HIPPOCAMPAL POPULATION BURSTS}

In addition to regular oscillations, the hippocampal network spontaneously generates population bursts at irregular intervals both during development and in the adult animal. Here we discuss the involvement of GABAergic inhibition in initiating these events and compare the mechanisms of engagement in developing and mature tissue.

\section{GIANT DEPOLARIZING POTENTIALS}

Already at a very young age the hippocampal CA3 region generates intermittent synchronous bursts. First described by Ben-Ari et al. (1989), CA3 hippocampal neurons in slices taken from immature rats, postnatal day (P) 0 to $\mathrm{P} 8$, spontaneously display brief episodes of depolarization of $25-50 \mathrm{mV}$, so called giant depolarizing potentials (GDPs), lasting 300-500 ms with an incidence of about 0.1 per second, and often accompanied by action potentials (Ben-Ari et al., 1989; Ben-Ari, 2001). Later, elegant in vivo studies confirmed the presence of such bursts early in development which were shown to share common features with their in vitro counterparts (Leinekugel et al., 2002). The intracellular depolarization was synchronous with bursts observed in field recordings, suggesting that they are the result of synchronous firing of a large population of neurons. GDPs were shown to depend on GABAergic transmission, as they could be blocked by a variety of $\mathrm{GABA}_{\mathrm{A}}$ receptor antagonists (Ben-Ari et al., 1989). Further evidence corroborating a role for GABAergic transmission in generating these events was the observation that the incidence of GDPs decreased from P5 onwards, and finally disappeared by P12, coinciding with the developmental transition from GABA exerting a depolarizing effect to being hyperpolarizing on a postsynaptic neuron. It has been suggested that the depolarizing effect of GABA is the result of an active $\mathrm{Na}^{+}-\mathrm{K}^{+}-\mathrm{Cl}^{-}$cotransporter (NKCC1) which sets up a $\mathrm{Cl}^{-}$gradient responsible for the depolarizing effect (Russell, 2000; Delpire and Mount, 2002). The ontogenetic shift to hyperpolarizing GABA action is caused by a concomitant developmental down-regulation of $\mathrm{NKCC} 1$ and an up-regulation of a $\mathrm{K}^{+}-\mathrm{Cl}^{-}$-cotransporter (KCC2) (Rivera et al., 1999).

Ben-Ari and colleagues suggest a mechanism by which activity of GABAergic neurons, spontaneous or induced by activation of their glutamatergic receptors, could depolarize populations of CA3 pyramidal neurons into generating such population bursts. Even though interneurons are more mature than pyramidal neurons at this stage (Soriano et al., 1986; Gozlan and Ben-Ari, 2003), the overall hippocampal circuitry and synaptic connections are still quite immature (Bahr and Wolff, 1985) with GABA being released mostly from axo-dendritic synapses or from non-synaptic free endings (Ben-Ari et al., 2004). It has been suggested that early network synchronization might have a role in controlling neuronal differentiation, synaptogenesis, and synaptic plasticity in the developing brain (Katz and Shatz, 1996; Khazipov and Luhmann, 2006; Blankenship and Feller, 2010).

\section{HUB NEURONS}

Far from being a homogeneous population of young immature interneurons, a recent study showed that not all inhibitory neurons are equal in the developing CA3 hippocampus. Already at such a 


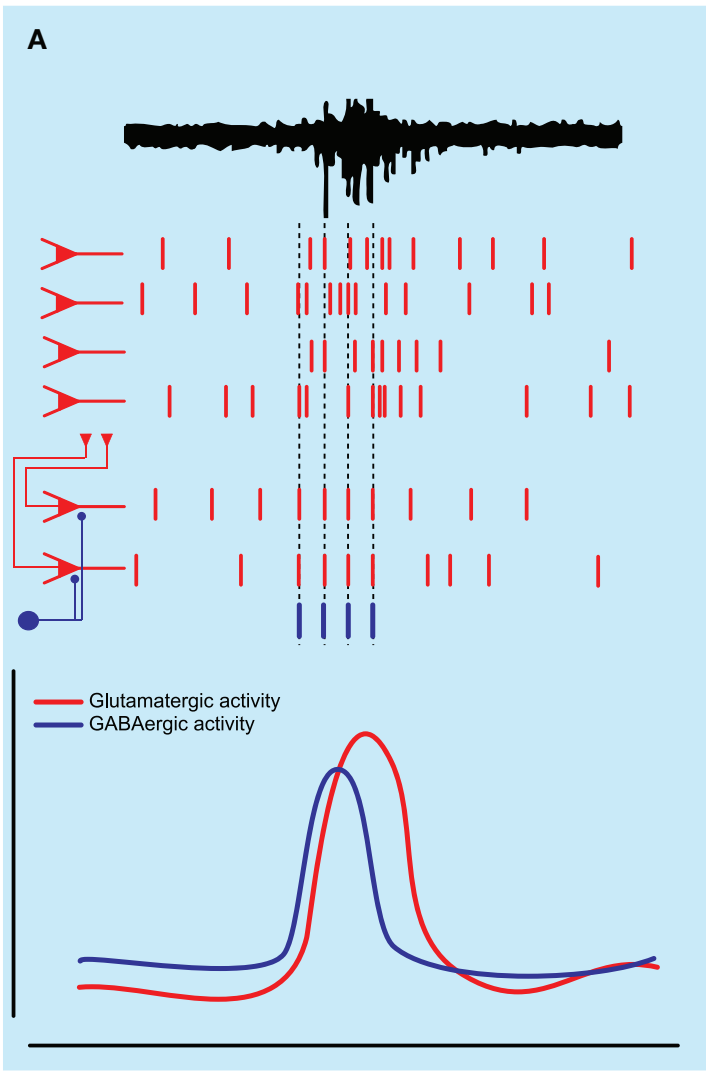

FIGURE 2 | Schematic diagram of network activity underlying hippocampal population bursts. (A) Spontaneous network synchronizations as seen in the immature hippocampus are thought to be generated by the excitatory action of GABAergic neurons, which depolarize and recruit a population of pyramidal neurons

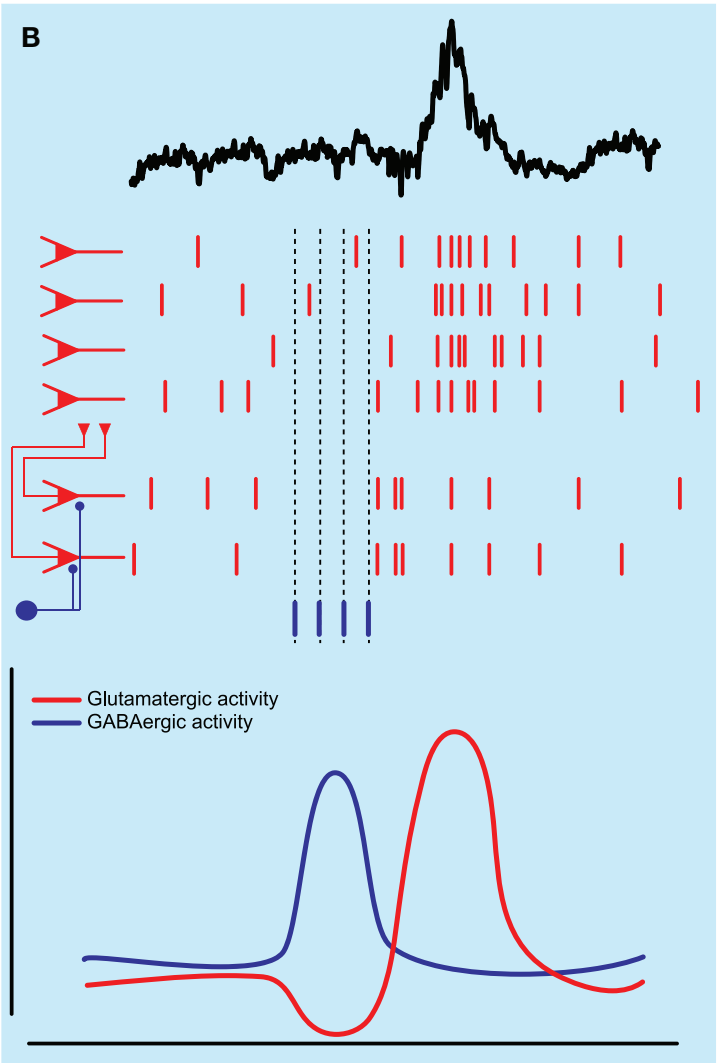

into a population burst. (B) Sharp wave-ripple population bursts as seen in the adult hippocampus may be initiated by the activity of an inhibitory interneuron temporarily silencing a subset of pyramidal neurons followed by rebound excitation, recruiting a larger population of pyramidal neurons into a population burst. young age they exhibit a functional heterogeneity, differentially affecting spontaneous network synchronization (Bonifazi et al., 2009). The authors of this study combined multibeam two-photon calcium imaging of ongoing network synchronization events (the field equivalent of intracellularly recorded GDPs) with stimulation of individual anatomically identified neurons in slices taken from both rat and GAD67-GFP mice. Spontaneous network synchronization was observed as synchronous $\mathrm{Ca}^{2+}$ signals in a large population of neurons (Leinekugel et al., 1997). Using this technique the authors showed that some neurons in the developing hippocampus have a very high functional connectivity to other neurons in the slice, which was detected online by finding neurons which were consistently active before other neurons. If a neuron was consistently active prior to $40 \%$ of the total number of imaged neurons it was deemed a high connectivity (HC) neuron. The authors then repeatedly depolarized individual HC neurons to supra-threshold levels with 200-ms long current pulses and showed that eight out of 20 of these HC neurons could significantly alter ongoing spontaneous network activity (see Figure 2A). They were dubbed "hub neurons" and, as hub neurons found in GAD67-GFP mice were all GFP positive and all hub neurons were aspiny, they were suggested to be GABAergic. The network response to activation of individual hub neurons was heterogeneous as they could silence the network, delay the generation of spontaneous network synchronization or initiate network synchronization events. Part of the heterogeneity in responses was explained by the observation of two different groups of GABAergic neuron based on axon arborization. Post hoc anatomical investigation of these neurons revealed that those with an axonal arborization close to the pyramidal cell layer, reminiscent of perisomatic targeting interneurons found in the adult hippocampus, were responsible for the initiation of network synchronization events (Bonifazi et al., 2009). Conversely, hub neurons with long projecting axons and sparse collaterals were responsible for silencing or delaying the generation of these events. This study is one of the first demonstrations of a small-scale network architecture present in the developing brain (Bullmore and Sporns, 2009) and raises several interesting points.

Firstly, it has been reported that the first GABAergic synapses are preferentially made on the apical dendrites and not on the soma of pyramidal neurons (Tyzio et al., 1999) with axosomatic targeting neurons only appearing later (Ben-Ari et al., 2004). In particular, it has been shown that certain basket cells initially form dendritic synapses and only shift to their adult pattern of somatic innervation after P4 (Morozov and Freund, 2003). This would suggest that an individual hub neuron responsible for the initiation of network synchronization events, even though anatomically resembling future perisomatic targeting interneurons, might innervate the dendrites of pyramidal neurons, which seems sufficient for spike generation in pyramidal neurons at this age (Ben-Ari et al., 1989). 
Secondly, a subset of HC neurons, shown to be pyramidal neurons, did not affect network activity suggesting that glutamatergic transmission is not sufficient to recruit large populations of neurons at this age.

Thirdly, the different responses found after activation of hub neurons might not only be the result of morphological differences between the two groups, possibly reflecting different developmental stages, but could also be influenced by the postsynaptic effect of GABA, since GABA might be both depolarizing and hyperpolarizing at this transitory phase of development.

In addition to the population of $\mathrm{HC}$ neurons there was a population of low connectivity (LC) neurons which had either a pyramidal neuron or interneuron morphology. These were classified according to the fact that their activity was not followed by any significant spontaneous population activity. Similarly, stimulating these neurons did not evoke any detectable changes in the network. The LC neurons were shown to have a shorter axonal length and this might explain their inability to synchronize a large population of neurons. As the immature hippocampus exhibits a high degree of heterogeneity, LC and $\mathrm{HC}$ neurons might actually represent the same types of neurons but at different stages in their development (de Lecea et al., 1995; Ben-Ari, 2001), or be differentially affected by the slicing procedure.

Lastly, it is unknown whether any further differences exist within the population of HC neurons or between the LC and HC neurons. Further investigation into the density, morphology, and location of synaptic contacts might be interesting.

It should be pointed out that both rat and mouse slices were used for these experiments (animals ranging in age from P5-P7) and it is at present unknown if there are developmental differences between the two rodent species in hub neuron function, reflecting differences in synapse development as well as differential transition between depolarizing and hyperpolarizing GABA action. It has recently been shown that there are both anatomical and electrophysiological differences between rat and mouse pyramidal neurons (Routh et al., 2009).

The paper by Bonifazi et al. raises the question whether neurons with similar properties exist in the adult hippocampus. A recent study by Ellender et al. (2010) would suggest that neurons with hub-like properties may exist also in the adult hippocampus.

\section{SHARP WAVE - RIPPLES}

At first it seems a daunting task to find hub neurons, bearing in mind the enormous diversity of interneurons in the adult hippocampus (Klausberger and Somogyi, 2008). Classifying the interneurons according to the location of their axonal targets already greatly facilitates the handling of such diversity (Freund and Buzsaki, 1996). Some of the hub neurons identified by Bonifazi et al., namely those that were able to initiate network synchronization events, had some morphological features similar to those described for the family of perisomatic targeting interneurons seen in the adult hippocampus, which can be subdivided into parvalbumin-positive basket cells (Kosaka et al., 1987), cholecystokinin-positive basket cells (Somogyi et al., 1984), and parvalbumin-positive axo-axonic cells (Somogyi, 1977; Somogyi et al., 1983). The different subtypes have been suggested to have different functions in the hippocampal network (Freund and Katona, 2007).
Similar to the very young hippocampus, the adult hippocampus (from P14 onwards) can also spontaneously generate bursts (Vanderwolf, 1969). These are called "ripples" (O'Keefe and Nadel, 1978) or sharp wave-ripple complexes (Buzsaki et al., 1983). Sharp wave-ripple complexes have two components. The sharp wave part is seen as a voltage deflection of around $1 \mathrm{mV}$ and $50-100 \mathrm{~ms}$ duration (Buzsaki, 1986). The second component, the so called ripple, is a fast oscillation with a frequency between 120 and $200 \mathrm{~Hz}$ (Buzsaki et al., 1992; Ylinen et al., 1995; Chrobak and Buzsaki, 1996). Sharp wave-ripple activity is seen during rest (e.g. awake immobility or eating) and slow-wave sleep. Sharp wave-ripples have been implicated in the reactivation of spike sequences (Skaggs and McNaughton, 1996; Lee and Wilson, 2002; Foster and Wilson, 2006; Diba and Buzsaki, 2007) and could facilitate the strengthening of memories within the hippocampus during exploration (O'Neill et al., 2006) as well as mediate off-line memory transfer to extra-hippocampal regions for long-term storage (Buzsaki, 1989). They are thought to be the result of synchronous bursts of action potentials in a subset of hippocampal pyramidal neurons (Buzsaki, 1986; Csicsvari et al., 2000), initiated in the CA3 subfield by pyramidal neurons with strong synaptic interconnections (Buzsaki and Chrobak, 1995) or recent place-related firing (O'Neill et al., 2006; Diba and Buzsaki, 2007). The detailed mechanism of their initiation in hippocampal CA3 is unknown, but an involvement of interneurons in shaping this network pattern appears likely.

Ellender et al. used hippocampal slices taken from rats aged P14P28, when neurons have axons and dendrites with adult-like features (Gaiarsa et al., 1992; Gomez-Di Cesare et al., 1997). Slices prepared at these ages can generate sharp wave-ripple activity spontaneously (Kubota et al., 2003). This model of sharp wave-ripples was adapted for submerged types of recording chamber (Hajos et al., 2009), which enabled visually guided patch-clamp recordings of neurons in CA3 with concomitant recording of ongoing sharp wave-ripple activity using planar multi-electrode arrays. The authors focused on the sharp waves and showed that they are associated with the synchronous firing of small changing populations of CA3 pyramidal neurons. Similar to the network synchronization events in developing hippocampus (Bonifazi et al. 2009), a high incidence of sharp waves was observed in region $\mathrm{CA} 3 \mathrm{c}$. The surrounding population of pyramidal neurons was inhibited; therefore, it was not surprising that blocking all $\mathrm{GABA}_{\mathrm{A}}$ receptor-mediated inhibition led to a transition from local sharp wave generation to large-scale epileptiform bursting (Miles and Wong, 1987). It was unexpected, however, that blocking only the phasic component of $\mathrm{GABA}_{\mathrm{A}}$ receptor-mediated inhibition completely and reversibly blocked all sharp wave generation (Ellender et al., 2010). This suggests that inhibition plays a previously unknown role in sharp wave initiation.

To investigate this further the authors performed whole-cell current-clamp recordings of anatomically identified neurons, which were repeatedly depolarized to supra-threshold levels with 500 -ms long current pulses every $10 \mathrm{~s}$, and observed the effect on network activity. Activation of either single pyramidal neurons or dendritic targeting interneurons did not affect network activity. In contrast, the activation of a subset of individual perisomatic targeting interneurons could both suppress (during activation), and subsequently enhance, the local generation of sharp waves. The authors investigated the mechanisms by which this subset 
of perisomatic targeting interneurons could initiate sharp wave generation by combining stimulation of individual perisomatic targeting interneurons with whole-cell voltage-clamp recording of a neighboring neuron. Voltage-clamped neurons were used to record both the excitatory and inhibitory activity in the network before, during, and after activation of the perisomatic targeting interneuron. It was shown that activity in perisomatic targeting interneurons was followed by a transient increase in excitation over inhibition in the network which preceded the initiation of a sharp wave. The authors concluded that this increase in excitation over inhibition could facilitate population burst generation by bringing a population of pyramidal neurons closer to threshold (Ellender et al., 2010).

This study suggests that GABA released by interneurons in the adult hippocampus can initiate population bursts, albeit via a different mechanism to that seen in the immature hippocampus. Instead of a direct depolarizing effect of GABA on pyramidal neurons, it is suggested that activity of perisomatic targeting interneurons temporarily silences a population of pyramidal neurons after which rebound excitation can lead to the synchronization of this population of pyramidal neurons (see Figure 2B). As mature rats were used, in which pyramidal cells most likely exhibit a $\mathrm{GABA}_{\mathrm{A}}$ receptor reversal potential negative to resting membrane potential (Luhmann and Prince, 1991; Owens et al., 1996), it was suggested that GABA would be hyperpolarizing. Nevertheless, it might be that GABA was excitatory in a subset of pyramidal neurons, as can be seen in young animals, as well as in certain neuronal types and cortical states in adult brain (Cohen et al., 2002; Gulledge and Stuart, 2003; Wozny et al., 2003; Banke and McBain, 2006; Szabadics et al., 2006), or that GABA could be depolarizing in specific subregions of the pyramidal neuron (e.g., axon initial segment; Szabadics et al., 2006; but see Glickfeld et al., 2009).

A subset of anatomically identified perisomatic targeting interneurons was not able to initiate sharp wave population bursts. The authors did not find significant differences between the successful and unsuccessful perisomatic targeting interneurons in either their average firing frequency, location within hippocampal CA3 or axonal length (Ellender et al., 2010). As no immunocytochemistry or electron microscopy was performed to further subdivide the population of perisomatic targeting interneurons it was not possible to determine whether the failure of some perisomatic targeting interneurons to influence sharp wave initiation is due to the network state or because different subclasses of perisomatic targeting interneuron might have different effects on these network events. Studies in area CA1 of the hippocampus in vivo have shown that parvalbumin-positive basket cells fire preferentially during a sharp wave-ripple, whereas axo-axonic cells tend to fire immediately before, but remain silent during sharp wave-ripples (Klausberger et al., 2003). It has not been reported whether these types of neurons in area CA3 show similar behaviors. If axo-axonic cells in CA3 also fire preferentially prior to sharp wave-ripples, they may have a role in selecting the subpopulation of CA3 pyramidal neurons that initiate a sharp wave event. Their preference to make synaptic connections on the axon initial segment of pyramidal neurons would make them well suited to influence axonal output (Miles et al., 1996).
It is well established that some fast spiking interneurons innervate themselves (Tamas et al., 1997). Furthermore, fast spiking interneurons are vastly interconnected through chemical and electrical synapses (Fukuda and Kosaka, 2000), both of which contribute to the synchronization of interneuronal networks (Tamas et al., 2000; Traub et al., 2001a; Whittington and Traub, 2003). It is at present unknown whether chemical synapses or electrical synapses (gap junctions) are necessary for the ability of single hub neurons or perisomatic targeting interneurons to induce hippocampal population bursts. Gap junctions might contribute to the generation of some network patterns (Maier et al., 2002; Buhl et al., 2003), but studies into their involvement are notoriously difficult as the presently available pharmacological agents are non-specific (Connors and Long, 2004).

The finding that single interneurons can facilitate the generation of hippocampal population bursts in both young and adult hippocampus is novel. The question arises whether the neurons found by Ellender et al., are mature versions of the hub neurons found by Bonifazi et al., or whether they are different types of neurons with similar hub-like properties. It might be that the hub neurons described by Bonifazi et al. are a transient population that disappears and dies later in development (Super et al., 1998). Molecular markers (such as parvalbumin or cholecystokinin) are used to classify interneurons in the adult hippocampus (Klausberger et al., 2003, 2004, 2005; Klausberger and Somogyi, 2008), and it would be interesting to investigate the expression of these markers in both the hub neurons and the successful perisomatic targeting interneurons. Such analysis, in combination with studies of ion channel and transcription factor expression (Cobos et al., 2006), might provide a fingerprint of the type of neuron capable of initiating network events, facilitating possible detection in other brain regions.

\section{EPILEPTIFORM BURSTS}

Lastly, we will briefly discuss the potential implications of the studies by Bonifazi et al. and Ellender et al. on our understanding of the mechanisms underlying epileptiform bursting. These two studies suggest that inhibition not only keeps population bursts in check (Trevelyan et al., 2007), but could also be actively involved in the initiation of population bursts. Classically, the generation of pathological epileptiform bursts is suggested to depend on a reduction of $\mathrm{GABA}_{\mathrm{A}}$ receptor-mediated inhibition, which facilitates mutual synaptic excitation (Traub and Wong, 1982). In systems where glutamatergic transmission is immature (Bonifazi et al., 2009) or embedded in a mature inhibitory network (Ellender et al., 2010), activity of individual pyramidal neurons was not sufficient to initiate population bursts. In contrast, a single CA3 pyramidal neuron can initiate an epileptiform burst in disinhibited conditions (Miles and Wong, 1983) by recruiting sufficient pyramidal neurons to exceed a threshold for burst initiation (Menendez de la Prida et al., 2006).

It has been shown in surgically removed human epileptic tissue that perisomatic inhibition is retained in the CA1 region of the hippocampus (Wittner et al., 2005) and retained or enhanced in the dentate gyrus (Isokawa-Akesson et al., 1989; Wittner et al., 2001). Wittner et al. observed an increase in inhibitory contacts at the axon initial segments of granule cells of the dentate gyrus. 
It was suggested that hyper-innervation of axon initial segments may lead to a more effective synchronization of granule cell firing and could in fact contribute to the generation or amplification of epileptic seizures (Wittner et al., 2001). More recently, it has been shown that nicotinic enhancement of GABA release can aggravate seizure generation in several models of autosomal dominant nocturnal frontal lobe epilepsy (Klaassen et al., 2006; Mann and Mody, 2008), further emphasizing the possibility that inhibition could facilitate pathological burst generation. It has also been shown that perisomatic targeting interneurons are massively recruited during epileptiform population bursts (Marchionni and Maccaferri, 2009).

Another possibility is that activity of perisomatic targeting interneurons is the last effort to keep a full burst at bay (Trevelyan et al., 2007; Trevelyan, 2009). In fact, it has been suggested that loss of dendritic inhibition could reduce seizure threshold, with preserved somatic inhibition keeping the network from a continuous occurrence of population bursts (Cossart et al., 2001). This would suggest that separate targeting of dendritic and perisomatic inhibition might be necessary for the effective treatment of epileptiform discharges (Magloczky and Freund, 2005). Lastly, it could also be that GABA swaps sides in pathology from being inhibitory to being excitatory due to a change in intracellular ion concentrations (Cohen et al., 2002).

In conclusion, both the studies by Bonifazi et al. (2009) and Ellender et al. (2010) imply that interneurons, rather than merely modulating pyramidal cell activity, can play an integral part in the local information processing that takes place in the hippocampal network. Furthermore, they suggest that neurons with hub-like properties exist in both the developing and mature hippocampus (Bullmore and Sporns, 2009) and corroborate the idea that single neurons can have a large effect on network activity (Brecht et al.,

\section{REFERENCES}

Alonso, A., and Llinas, R. R. (1989). Subthreshold Na+-dependent thetalike rhythmicity in stellate cells of entorhinal cortex layer II. Nature 342, 175-177.

Ayala, G. F., Dichter, M., Gumnit, R. J., Matsumoto, H., and Spencer, W. A. (1973). Genesis of epileptic interictal spikes. New knowledge of cortical feedback systems suggests a neurophysiological explanation of brief paroxysms. Brain Res. 52, 1-17.

Bahr, S., and Wolff, J. R. (1985). Postnatal development of axosomatic synapses in the rat visual cortex: morphogenesis and quantitative evaluation. J. Comp. Neurol. 233, 405-420.

Banke, T. G., and McBain, C. J. (2006). GABAergic input onto CA3 hippocampal interneurons remains shunting throughout development. J. Neurosci. 26, 11720-11725.

Bazelot, M., Dinocourt, C., Cohen, I., and Miles, R. (2010). Unitary inhibitory field potentials in the CA3 region of rat hippocampus. J. Physiol. 588, 2077-2090.
Ben-Ari, Y. (2001). Developing networks play a similar melody. Trends Neurosci. 24, 353-360.

Ben-Ari, Y., Cherubini, E., Corradetti, R., and Gaiarsa, J. L. (1989). Giant synaptic potentials in immature rat CA3 hippocampal neurones. J. Physiol. 416, 303-325.

Ben-Ari, Y., Khalilov, I., Represa, A., and Gozlan, H. (2004). Interneurons set the tune of developing networks. Trends Neurosci. 27, 422-427.

Blankenship,A.G., and Feller,M.B. (2010). Mechanisms underlying spontaneous patterned activity in developing neural circuits. Nat. Rev. Neurosci. 11, 18-29.

Bonifazi, P., Goldin, M., Picardo, M. A., Jorquera, I., Cattani, A., Bianconi, G., Represa, A., Ben-Ari, Y., and Cossart, R. (2009). GABAergic hub neurons orchestrate synchrony in developing hippocampal networks. Science 326, 1419-1424.

Bragin, A., Jando, G., Nadasdy, Z., Hetke, J., Wise, K., and Buzsaki, G. (1995). Gamma $(40-100 \mathrm{~Hz})$ oscillation in the hippocampus of the behaving rat. $J$. Neurosci. 15, 47-60.

2004; Houweling and Brecht, 2008; Li et al., 2009). The finding that interneurons can initiate physiological hippocampal population bursts raises the possibility that they might also contribute to those seen in pathology.

\section{FUTURE}

The studies discussed here extend the roles of perisomatic inhibition beyond that of rhythm generation, showing that they can also contribute to the transmembrane currents underlying hippocampal field oscillations and are able to initiate hippocampal population bursts. It remains to be seen whether these observations hold for network activity in vivo. However, as these observations were made in slice preparations, in which axonal projections are markedly reduced, one might expect that their ability to generate transmembrane currents, as well as influence population burst activity, might be even greater in vivo.

With the development of in vivo recording techniques which enable monitoring extracellular activity early in development (Yang et al., 2009), as well as intracellularly in anatomically identified neurons in behaving animals (Epsztein et al., 2010) combined with optogenetics (Deisseroth et al., 2006) and modeling, in vivo investigation of these findings will be possible. By gaining a better understanding of the cellular mechanisms involved in network activity one may hope that their functions in cognitive processes will be uncovered, and such understanding might also help us in combating brain disorders (Uhlhaas and Singer, 2006, 2010; Hammond et al., 2007).

\section{ACKNOWLEDGMENTS}

We would like to thank Wiebke Nissen for careful reading of a draft of this review. The authors' research was supported by The Wellcome Trust.

Bragin, A., Mody, I., Wilson, C. L., and Engel, J. Jr. (2002). Local generation of fast ripples in epileptic brain. $J$. Neurosci. 22, 2012-2021.

Bragin, A., Wilson, C. L., and Engel, J. Jr. (2000). Chronic epileptogenesis requires development of a network of pathologically interconnected neuron clusters: a hypothesis. Epilepsia 41(Suppl. 6), S144-S152.

Bragin, A., Wilson, C. L., and Engel, J. Jr. (2007). Voltage depth profiles of high-frequency oscillations after kainic acid-induced status epilepticus. Epilepsia 48(Suppl. 5), 35-40.

Brecht, M., Schneider, M., Sakmann, B. and Margrie, T. W. (2004). Whisker movements evoked by stimulation of single pyramidal cells in rat motor cortex. Nature 427, 704-710.

Brunel, N., and Wang, X. J. (2003). What determines the frequency of fast network oscillations with irregular neural discharges? I. Synaptic dynamics and excitation-inhibition balance. $J$. Neurophysiol. 90, 415-430.

Buhl, D. L., and Buzsaki, G. (2005). Developmental emergence of hippoc- ampal fast-field "ripple" oscillations in the behaving rat pups. Neuroscience 134, 1423-1430.

Buhl, D. L., Harris, K. D., Hormuzdi, S. G., Monyer, H., and Buzsaki, G. (2003). Selective impairment of hippocampal gamma oscillations in connexin-36 knock-out mouse in vivo. J. Neurosci. 23, 1013-1018.

Bullmore, E., and Sporns, O. (2009). Complex brain networks: graph theoretical analysis of structural and functional systems. Nat. Rev. Neurosci. 10, 186-198.

Buzsaki, G. (1986). Hippocampal sharp waves: their origin and significance. Brain Res. 398, 242-252.

Buzsaki, G. (1989). Two-stage model of memory trace formation: a role for "noisy" brain states. Neuroscience 31, 551-570.

Buzsaki, G. (2002). Theta oscillations in the hippocampus. Neuron 33, 325-340.

Buzsaki, G., Buhl, D. L., Harris, K. D. Csicsvari, J., Czeh, B., and Morozov, A. (2003). Hippocampal network patterns of activity in the mouse. Neuroscience 116, 201-211. 
Buzsaki, G., and Chrobak, J. J. (1995). Temporal structure in spatially organized neuronal ensembles: a role for interneuronal networks. Curr. Opin. Neurobiol. 5, 504-510.

Buzsaki, G., Horvath,Z., Urioste, R., Hetke, J., and Wise, K. (1992).High-frequency network oscillation in the hippocampus. Science 256, 1025-1027.

Buzsaki, G., Leung, L. W., and Vanderwolf, C. H. (1983). Cellular bases of hippocampal EEG in the behaving rat. Brain Res. 287, 139-171.

Chrobak, J. J., and Buzsaki, G. (1996). High-frequency oscillations in the output networks of the hippocampalentorhinal axis of the freely behaving rat. J. Neurosci. 16, 3056-3066.

Cobb, S. R., Buhl, E. H., Halasy, K., Paulsen, O., and Somogyi, P. (1995). Synchronization of neuronal activity in hippocampus by individual GABAergic interneurons. Nature 378, 75-78.

Cobos, I., Long, J. E., Thwin, M. T., and Rubenstein, J. L. (2006). Cellular patterns of transcription factor expression in developing cortical interneurons. Cereb. Cortex 16(Suppl. 1), i82-i88.

Cohen, I., Navarro, V., Clemenceau, S. Baulac, M., and Miles, R. (2002). On the origin of interictal activity in human temporal lobe epilepsy in vitro. Science 298, 1418-1421.

Connors, B. W., and Long, M. A. (2004). Electrical synapses in the mammalian brain. Annu. Rev. Neurosci. 27, 393-418.

Cossart, R., Dinocourt, C., Hirsch, J. C., Merchan-Perez, A., De Felipe, J., BenAri, Y., Esclapez, M., and Bernard, C. (2001). Dendritic but not somatic GABAergic inhibition is decreased in experimental epilepsy. Nat. Neurosci. 4, 52-62.

Csicsvari, J., Hirase, H., Czurko, A., Mamiya, A., and Buzsaki, G. (1999). Fast network oscillations in the hippocampalCA1 region of the behaving rat. J. Neurosci. 19:RC20.

Csicsvari, J., Hirase, H., Mamiya, A., and Buzsaki, G. (2000). Ensemble patterns of hippocampal CA3-CA1 neurons during sharp wave-associated population events. Neuron 28, 585-594.

Csicsvari, J., Jamieson, B., Wise, K. D., and Buzsaki, G. (2003). Mechanisms of gamma oscillations in the hippocampus of the behaving rat. Neuron 37 , 311-322.

Deisseroth, K., Feng, G., Majewska, A. K., Miesenbőck, G., Ting, A., and Schnitzer, M. J. (2006). Next generation optical technologies for illuminating genetically targeted brain circuits. J. Neurosci. 26, 10380-10386. de Lecea, L., del Rio, J. A., and Soriano, E. (1995). Developmental expression of parvalbumin mRNA in the cerebral cortex and hippocampus of the rat. Brain Res. Mol. Brain Res. 32, 1-13.

Delpire, E., and Mount, D. B. (2002). Human and murine phenotypes associated with defects in cation-chloride cotransport. Annu. Rev. Physiol. 64, 803-843.

Diba, K., and Buzsaki, G. (2007). Forward and reverse hippocampal placecell sequences during ripples. Nat. Neurosci. 10, 1241-1242.

Draguhn, A., Traub, R. D., Schmitz, D., and Jefferys, J. G. (1998). Electrical coupling underlies high-frequency oscillations in the hippocampus in vitro. Nature 394, 189-192.

Dzhala, V. I., and Staley, K. J. (2004). Mechanisms of fast ripples in the hippocampus. J. Neurosci. 24, 8896-8906.

Ellender, T. J., Nissen, W., Colgin, L. L., Mann, E. O., and Paulsen, O. (2010). Priming of hippocampal population bursts by individual perisomatictargeting interneurons. J. Neurosci. 30, 5979-5991.

Epsztein, J., Lee, A. K., Chorev, E., and Brecht, M. (2010). Impact of spikelets on hippocampal CA1 pyramidal cell activity during spatial exploration. Science 327, 474-477.

Fisahn, A., Pike, F. G., Buhl, E. H., and Paulsen, O. (1998). Cholinergic induction of network oscillations at $40 \mathrm{~Hz}$ in the hippocampus in vitro. Nature 394, 186-189.

Foffani, G., Uzcategui, Y. G., Gal, B., and Menendez de la Prida, L. (2007). Reduced spike-timing reliability correlates with the emergence of fast ripples in the rat epileptic hippocampus. Neuron 55, 930-941.

Foster, D. J., and Wilson, M. A. (2006). Reverse replay of behavioural sequences in hippocampal place cells during the awake state. Nature 440 680-683.

Freund, T. F., and Buzsaki, G. (1996). Interneurons of the hippocampus. Hippocampus 6, 347-470.

Freund, T. F., and Katona, I. (2007) Perisomatic inhibition. Neuron 56, 33-42.

Fukuda, T., and Kosaka, T. (2000). Gap junctions linking the dendritic network of GABAergic interneurons in the hippocampus. J. Neurosci. 20, 1519-1528.

Gaiarsa, J. L., Beaudoin, M., and Ben-Ari, Y. (1992). Effect of neonatal degranulation on the morphological development of rat CA3 pyramidal neurons: inductive role of mossy fibers on the formation of thorny excrescences. $J$. Comp. Neurol. 321, 612-625.
Glickfeld, L. L., Roberts, J. D., Somogyi, P., and Scanziani, M. (2009). Interneurons hyperpolarize pyramidal cells along their entire somatodendritic axis. Nat. Neurosci. 12, 21-23.

Gomez-Di Cesare, C. M., Smith, K. L. Rice, F. L., and Swann, J. W. (1997) Axonal remodeling during postnatal maturation of CA3 hippocampal pyramidal neurons. J. Comp. Neurol. 384, 165-180.

Goutagny, R., Jackson, J., and Williams, S. (2009). Self-generated theta oscillations in the hippocampus. Nat. Neurosci. 12, 1491-1493.

Gozlan, H., and Ben-Ari, Y. (2003) Interneurons are the source and the targets of the first synapses formed in the rat developing hippocampal circuit. Cereb. Cortex 13, 684-692.

Grenier, F., Timofeev, I., and Steriade, M. (2003). Neocortical very fast oscillations (ripples, $80-200 \mathrm{~Hz}$ ) during seizures: intracellular correlates. $J$. Neurophysiol. 89, 841-852.

Gulledge, A. T., and Stuart, G. J. (2003) Excitatory actions of GABA in the cortex. Neuron 37, 299-309.

Hajos, N., Ellender, T. J., Zemankovics, R., Mann, E. O., Exley, R., Cragg, S. J., Freund, T. F., and Paulsen, O. (2009). Maintaining network activity in submerged hippocampal slices: importance of oxygen supply. Eur. J. Neurosci. 29, 319-327.

Hajos, N., Palhalmi, J., Mann, E. O., Nemeth, B., Paulsen, O., and Freund, T. F. (2004). Spike timing of distinct types of GABAergic interneuron during hippocampal gamma oscillations in vitro. J. Neurosci. 24 9127-9137.

Hajos, N., and Paulsen, O. (2009) Network mechanisms of gamma oscillations in the CA3 region of the hippocampus. Neural. Netw. 22, 1113-1119.

Hammond, C., Bergman, H., and Brown, P. (2007). Pathological synchronization in Parkinson's disease: networks, models and treatments. Trends Neurosci. 30, 357-364.

Houweling, A. R., and Brecht, M. (2008) Behavioural report of single neuron stimulation in somatosensory cortex. Nature 451, 65-68.

Huxter, J., Burgess, N., and O'Keefe, J. (2003). Independent rate and temporal coding in hippocampal pyramidal cells. Nature 425, 828-832.

Isokawa-Akesson, M., Wilson, C. L. and Babb, T. L. (1989). Inhibition in synchronously firing human hippocampal neurons. Epilepsy Res. 3 236-247.

Jensen, O., and Lisman, J.E. (1996). Theta/ gamma networks with slow NMDA channels learn sequences and encode episodic memory: role of NMDA channels in recall. Learn. Mem. 3 , 264-278.

Katsumaru, H., Kosaka, T., Heizmann, C. W., and Hama, K. (1988). Gap junctions on GABAergic neurons containing the calcium-binding protein parvalbumin in the rat hippocampus (CA1 region). Exp. Brain Res. 72, 363-370.

Katz, L. C., and Shatz, C. J. (1996). Synaptic activity and the construction of cortical circuits. Science 274, 1133-1138.

Khalilov, I., Le Van Quyen, M., Gozlan, H., and Ben-Ari, Y. (2005). Epileptogenic actions of GABA and fast oscillations in the developing hippocampus. Neuron 48, 787-796.

Khazipov, R., and Luhmann, H. J. (2006). Early patterns of electrical activity in the developing cerebral cortex of humans and rodents. Trends Neurosci. 29, 414-418.

Klaassen, A., Glykys, J., Maguire, J., Labarca, C., Mody, I., and Boulter, J. (2006). Seizures and enhanced cortical GABAergic inhibition in two mouse models of human autosomal dominant nocturnal frontal lobe epilepsy. Proc. Natl. Acad. Sci. U.S.A. 103, 19152-19157.

Klausberger, T. (2009). GABAergic interneurons targeting dendrites of pyramidal cells in the CA1 area of the hippocampus. Eur. J. Neurosci. 30, 947-957.

Klausberger, T., Magill, P. J., Marton, L. F., Roberts, J. D., Cobden, P. M., Buzsaki, G., and Somogyi, P. (2003). Brainstate- and cell-type-specific firing of hippocampal interneurons in vivo. Nature 421, 844-848.

Klausberger, T., Marton, L. F., Baude, A., Roberts, J. D., Magill, P. J., and Somogyi, P. (2004). Spike timing of dendrite-targeting bistratified cells during hippocampal network oscillations in vivo. Nat. Neurosci. 7, 41-47.

Klausberger, T., Marton, L. F., O’Neill, J., Huck, J. H., Dalezios, Y., Fuentealba, P., Suen, W. Y., Papp, E., Kaneko, T., Watanabe, M., Csicsvari, J., and Somogyi, P. (2005). Complementary roles of cholecystokinin- and parvalbumin-expressing GABAergic neurons in hippocampal network oscillations. J. Neurosci. 25, 9782-9793.

Klausberger, T., and Somogyi, P. (2008). Neuronal diversity and temporal dynamics: the unity of hippocampal circuit operations. Science 321 , 53-57.

Kosaka, T., Katsumaru, H., Hama, K., Wu, J. Y., and Heizmann, C. W. (1987). GABAergic neurons containing the $\mathrm{Ca}^{2+}$-binding protein parvalbumin in the rat hippocampus and dentate gyrus. Brain Res. 419, 119-130. 
Kubota, D., Colgin, L. L., Casale, M., Brucher, F. A., and Lynch, G. (2003). Endogenous waves in hippocampal slices. J. Neurophysiol. 89, 81-89.

Lee, A. K., and Wilson, M. A. (2002). Memory of sequential experience in the hippocampus during slow wave sleep. Neuron 36, 1183-1194.

Leinekugel, X., Khazipov, R., Cannon, R., Hirase, H., Ben-Ari, Y., and Buzsaki, G. (2002). Correlated bursts of activity in the neonatal hippocampus in vivo. Science 296, 2049-2052.

Leinekugel, X., Medina, I., Khalilov, I., Ben-Ari, Y., and Khazipov, R. (1997). $\mathrm{Ca}^{2+}$ oscillations mediated by the synergistic excitatory actions of GABA(A) and NMDA receptors in the neonatal hippocampus. Neuron 18, 243-255.

Le Van Quyen, M., Khalilov, I., and Ben-Ari, Y. (2006). The dark side of high-frequency oscillations in the developing brain. Trends Neurosci. 29, 419-427.

Leung, L. S., and Yim, C. Y. (1986). Intracellular records of theta rhythm in hippocampal CA1 cells of the rat. Brain Res. 367, 323-327.

Li, C. Y., Poo, M. M., and Dan, Y. (2009). Burst spiking of a single cortical neuron modifies global brain state. Science 324, 643-646.

Lisman, J. E., and Idiart, M. A. (1995). Storage of $7 \pm 2$ short-term memories in oscillatory subcycles. Science 267, 1512-1515.

Luhmann, H. J., and Prince, D. A. (1991). Postnatal maturation of the GABAergic system in rat neocortex. J. Neurophysiol. 65, 247-263.

Magloczky, Z., and Freund, T. F. (2005). Impaired and repaired inhibitory circuits in the epileptic human hippocampus. Trends Neurosci. 28, 334-340.

Maier, N., Guldenagel, M., Sohl, G., Siegmund, H., Willecke, K., and Draguhn, A. (2002). Reduction of high-frequency network oscillations (ripples) and pathological network discharges in hippocampal slices from connexin 36-deficient mice. J. Physiol. (Lond.) 541, 521-528.

Mann, E. O., and Mody, I. (2008). The multifaceted role of inhibition in epilepsy: seizure-genesis through excessive GABAergic inhibition in autosomal dominant nocturnal frontal lobe epilepsy. Curr. Opin. Neurol. 21, 155-160.

Mann, E. O., Suckling, J. M., Hajos, N., Greenfield, S. A., and Paulsen, O. (2005). Perisomatic feedback inhibition underlies cholinergically induced fast network oscillations in the rat hippocampus in vitro. Neuron 45, 105-117.

Marchionni, I., and Maccaferri, G. (2009). Quantitative dynamics and spatial profile of perisomatic GABAergic input during epileptiform synchronization in the CA1 hippocampus. $J$. Physiol. (Lond.) 587, 5691-5708.

Menendez de la Prida, L., Huberfeld, G., Cohen, I., and Miles, R. (2006). Threshold behavior in the initiation of hippocampal population bursts. Neuron 49, 131-142.

Miles, R., Toth, K., Gulyas, A. I., Hajos, N., and Freund, T. F. (1996). Differences between somatic and dendritic inhibition in the hippocampus. Neuron 16, 815-823.

Miles, R., and Wong, R. K. (1983). Single neurones can initiate synchronized population discharge in the hippocampus. Nature 306, 371-373.

Miles, R., and Wong, R. K. (1987). Inhibitory control of local excitatory circuits in the guinea-pig hippocampus. J. Physiol. (Lond.) 388, 611-629.

Morozov, Y. M., and Freund, T. F. (2003). Postnatal development and migration of cholecystokinin-immunoreactive interneurons in rat hippocampus. Neuroscience 120, 923-939.

O’Keefe, J., and Dostrovsky, J. (1971). The hippocampus as a spatial map. Preliminary evidence from unit activity in the freely-moving rat. Brain Res. 34, 171-175

O'Keefe, J., and Nadel, L. (1978). The Hippocampus as a Cognitive Map. Oxford: Oxford University Press.

O’Neill, J., Senior, T., and Csicsvari, J. (2006). Place-selective firing of CA1 pyramidal cells during sharp wave/ ripple network patterns in exploratory behavior. Neuron 49, 143-155.

Oren, I., Hajos, N., and Paulsen, O. (2010). Identification of the current generator underlying cholinergically induced gamma frequency field potential oscillations in the hippocampalCA3 region. J. Physiol. (Lond.) 588, 785-797.

Oren, I., Mann, E. O., Paulsen, O., and Hajos, N. (2006). Synaptic currents in anatomically identified CA3 neurons during hippocampal gamma oscillations in vitro. J. Neurosci. 26, 9923-9934.

Owens, D. F., Boyce, L. H., Davis, M. B., and Kriegstein, A. R. (1996). Excitatory GABA responses in embryonic and neonatal cortical slices demonstrated by gramicidin perforated-patch recordings and calcium imaging. $J$. Neurosci. 16, 6414-6423.

Penttonen, M., Kamondi, A., Acsady, L., and Buzsaki, G. (1998). Gamma frequency oscillation in the hippocampus of the rat: intracellular analysis in vivo. Eur. J. Neurosci. 10, 718-728.

Petsche, H., and Stumpf, C. (1962). [The origin of theta-rhytm in the rabbit hippocampus.]. Wien Klin Wochenschr 74, 696-700.
Rivera, C., Voipio, J., Payne, J. A., Ruusuvuori, E., Lahtinen, H., Lamsa, K., Pirvola, U., Saarma, M., and Kaila, K. (1999). The $\mathrm{K}^{+} / \mathrm{Cl}$ - co-transporter KCC2 renders GABA hyperpolarizing during neuronal maturation. Nature 397, 251-255.

Routh, B. N., Johnston, D., Harris, K., and Chitwood, R. A. (2009). Anatomical and electrophysiological comparison of CA1 pyramidal neurons of the rat and mouse. J. Neurophysiol. 102, 2288-2302.

Russell, J. M. (2000). Sodium-potassiumchloride cotransport. Physiol. Rev. 80, 211-276.

Skaggs, W. E., and McNaughton, B. L. (1996). Replay of neuronal firing sequences in rat hippocampus during sleep following spatial experience. Science 271, 1870-1873.

Soltesz, I., and Deschenes, M. (1993). Low- and high-frequency membrane potential oscillations during theta activity in CA1 and CA3 pyramidal neurons of the rat hippocampus under ketamine-xylazine anesthesia. J. Neurophysiol. 70, 97-116.

Somogyi, P. (1977). A specific "axoaxonal" interneuron in the visual cortex of the rat. Brain Res. 136 , 345-350.

Somogyi, P., Hodgson, A. J., Smith, A. D., Nunzi, M. G., Gorio, A., and Wu, J. Y. (1984). Different populations of GABAergic neurons in the visual cortex and hippocampus of cat contain somatostatin- or cholecystokininimmunoreactive material. J. Neurosci. 4, 2590-2603.

Somogyi, P., Nunzi, M. G., Gorio, A. and Smith, A. D. (1983). A new type of specific interneuron in the monkey hippocampus forming synapses exclusively with the axon initial segments of pyramidal cells. Brain Res. 259, 137-142.

Soriano, E., Cobas, A., and Fairen, A. (1986). Asynchronism in the neurogenesis of GABAergic and nonGABAergic neurons in the mouse hippocampus. Brain Res. 395 88-92.

Stewart, M., and Fox, S. E. (1990). Do septal neurons pace the hippocampal theta rhythm? Trends Neurosci. 13, 163-168.

Strata, F. (1998). Intrinsic oscillations in CA3 hippocampal pyramids: physiological relevance to theta rhythm generation. Hippocampus 8 , 666-679.

Super, H., Martinez, A., Del Rio, J. A. and Soriano, E. (1998). Involvement of distinct pioneer neurons in the formation of layer-specific connections in the hippocampus. J. Neurosci. 18, 4616-4626.
Szabadics, J., Varga, C., Molnar, G., Olah, S., Barzo, P., and Tamas, G. (2006). Excitatory effect of GABAergic axoaxonic cells in cortical microcircuits. Science 311, 233-235.

Tamas, G., Buhl, E. H., Lorincz, A. and Somogyi, P. (2000). Proximally targeted GABAergic synapses and gap junctions synchronize cortical interneurons. Nat. Neurosci. 3, 366-371.

Tamas, G., Buhl, E. H., and Somogyi, P. (1997). Massive autaptic selfinnervation of GABAergic neurons in cat visual cortex. J. Neurosci. 17, 6352-6364.

Traub, R.D., Jefferys, J.G., and Whittington, M. A. (1997). Simulation of gamma rhythms in networks of interneurons and pyramidal cells. J. Comput. Neurosci. 4, 141-150.

Traub, R. D., Kopell, N., Bibbig, A., Buhl, E. H., LeBeau, F. E., and Whittington, M.A.(2001a). Gap junctions between interneuron dendrites can enhance synchrony of gamma oscillations in distributed networks. J. Neurosci. 21, 9478-9486.

Traub, R. D., Whittington, M. A., Buhl, E. H., LeBeau, F. E., Bibbig, A., Boyd, S., Cross, H., and Baldeweg, T. (2001b). A possible role for gap junctions in generation of very fast EEG oscillations preceding the onset of, and perhaps initiating, seizures. Epilepsia 42, 153-170.

Traub, R. D., and Wong, R. K. (1982). Cellular mechanism of neuronal synchronization in epilepsy. Science 216, 745-747.

Trevelyan, A. J. (2009). The direct relationship between inhibitory currents and local field potentials. J. Neurosci. 29, 15299-15307.

Trevelyan, A. J., Sussillo, D., Watson, B. O., and Yuste, R. (2006). Modular propagation of epileptiform activity: evidence for an inhibitory veto in neocortex. J. Neurosci. 26, 12447-12455.

Trevelyan, A. J., Sussillo, D., and Yuste, R. (2007). Feedforward inhibition contributes to the control of epileptiform propagation speed. J. Neurosci. 27, 3383-3387.

Tukker, J. J., Fuentealba, P., Hartwich, K., Somogyi, P., and Klausberger, T. (2007). Cell type-specific tuning of hippocampal interneuron firing during gamma oscillations in vivo. $J$. Neurosci. 27, 8184-8189.

Tyzio, R., Represa, A., Jorquera, I., BenAri, Y., Gozlan, H., and Aniksztejn, L. (1999). The establishment of GABAergic and glutamatergic synapses on CAl pyramidal neurons is sequential and correlates with the development of the apical dendrite. J. Neurosci. 19, 10372-10382. 
Uhlhaas, P. J., and Singer,W. (2006). Neural synchrony in brain disorders: relevance for cognitive dysfunctions and pathophysiology. Neuron 52, 155-168.

Uhlhaas, P. J., and Singer, W. (2010). Abnormal neural oscillations and synchrony in schizophrenia. Nat. Rev. Neurosci. 11, 100-113.

Vanderwolf, C. H. (1969). Hippocampal electrical activity and voluntary movement in the rat. Electroencephalog.r Clin. Neurophysiol. 26, 407-418.

Vizi, E. S., and Kiss, J. P. (1998). Neurochemistry and pharmacology of the major hippocampal transmitter systems: synaptic and nonsynaptic interactions. Hippocampus 8, 566-607.

Wang, X.-J., and Buzsaki, G. (1996). Gamma oscillation by synaptic inhibition in a hippocampal interneuronal network model. J. Neurosci. 16, 6402-6413.
Whittington, M. A., and Traub, R. D. (2003). Interneuron diversity series: inhibitory interneurons and network oscillations in vitro. Trends Neurosci. 26, 676-682.

Whittington, M. A., Traub, R. D., and Jefferys, J.G. (1995). Synchronized oscillations in interneuron networks driven by metabotropic glutamate receptor activation. Nature 373, 612-615.

Wittner, L., Eross, L., Czirjak, S., Halasz, P., Freund, T.F., and Magloczky, Z. (2005). Surviving CA1 pyramidal cells receive intact perisomatic inhibitory input in the human epileptic hippocampus. Brain 128, 138-152.

Wittner, L., Magloczky, Z., Borhegyi, Z., Halasz, P., Toth, S., Eross, L., Szabo, Z., and Freund, T. F. (2001). Preservation of perisomatic inhibitory input of granule cells in the epileptic human dentate gyrus. Neuroscience 108, 587-600.
Wozny, C., Kivi, A., Lehmann, T. N., Dehnicke, C., Heinemann, U., and Behr, J. (2003). Comment on "On the origin of interictal activity in human temporal lobe epilepsy in vitro". Science 301:463; author reply 463.

Yang, J. W., Hanganu-Opatz, I. L., Sun, J. J., and Luhmann, H. J. (2009) Three patterns of oscillatory activity differentially synchronize developing neocortical networks in vivo. $J$. Neurosci. 29, 9011-9025.

Ylinen, A., Bragin, A., Nadasdy, Z., Jando, G., Szabo, I., Sik, A., and Buzsaki, G. (1995). Sharp wave-associated highfrequency oscillation $(200 \mathrm{~Hz})$ in the intact hippocampus: network and intracellular mechanisms. J. Neurosci. $15,30-46$.

Conflict of Interest Statement: The authors declare that the research was con- ducted in the absence of any commercial or financial relationships that could be construed as a potential conflict of interest.

Received: 18 March 2010; paper pending published: 26 April 2010; accepted: 28 June 2010; published online: $30 \mathrm{July}$ 2010.

Citation: Ellender TJ and Paulsen O (2010)

The many tunes of perisomatic targeting interneurons in the hippocampal network. Front. Cell. Neurosci. 4:26. doi: 10.3389/ fncel.2010.00026

Copyright (c) 2010 Ellender and Paulsen. This is an open-access article subject to an exclusive license agreement between the authors and the Frontiers Research Foundation, which permits unrestricted use, distribution, and reproduction in any medium, provided the original authors and source are credited. 